\title{
Eficácia do tratamento cognitivo e/ou comportamental para o transtorno de ansiedade social
}

\author{
Effectiveness of cognitive and/or behavioral therapy for social anxiety disorder
}

\author{
Sara Costa Cabral Mululo ${ }^{1}$, Gabriela Bezerra de Menezes $^{2}$, Leonardo Fontenelle ${ }^{3}$, Marcio Versiani ${ }^{4}$ \\ ${ }^{1}$ Graduada, Psicologia. Mestranda, Programa de Ansiedade e Depressão, Instituto de Psiquiatria, Universidade Federal do Rio de Janeiro (UFRJ), Rio de Janeiro, RJ. ${ }^{2}$ Doutora, \\ Psiquiatria. Pesquisadora, Programa de Ansiedade e Depressão, Instituto de Psiquiatria, UFRJ. ${ }^{3}$ Doutor, Psiquiatria. Pesquisador, Programa de Ansiedade e Depressão, Instituto \\ de Psiquiatria, UFRJ. ${ }^{4}$ Doutor, Psiquiatria. Diretor, Professor titular e Pesquisador, Programa de Ansiedade e Depressão, Instituto de Psiquiatria, UFRJ. \\ Trabalho realizado no Programa de Pesquisa em Ansiedade e Depressão, Instituto de Psiquiatria, Universidade Federal do Rio de Janeiro (UFRJ), Rio de Janeiro, RJ.
}

\section{Resumo}

Objetivo: Avaliar a eficácia do tratamento cognitivo e/ou comportamental no transtorno de ansiedade social quando comparado a outras intervenções terapêuticas, incluindo farmacoterapia.

Método: Realizamos análise sistemática de todos os ensaios clínicos controlados randomizados envolvendo terapia cognitiva e/ou comportamental versus outras formas de tratamento, publicados até março de 2009, indexados nas seguintes bases de dados: MEDLINE, PsycINFO, Registro Cochrane de Ensaios Controlados, LILACS e ISI/Web of Science.

Resultados: Os estudos avaliados não demonstraram diferenças significativas entre a terapia cognitiva e/ou comportamental e a farmacoterapia. Esses tratamentos combinados não apresentaram maior eficácia do que as intervenções isoladamente. Quando comparada ao grupo controle, a terapia cognitiva e/ou comportamental é eficaz na redução dos sintomas de ansiedade social, tanto no seu formato padrão quanto nas novas formas de intervenção. A exceção foi o treino em habilidades sociais, que, isoladamente, não se mostrou eficaz. A terapia cognitiva e/ou comportamental também demonstrou ser superior aos outros tipos de psicoterapias.

Conclusão: A terapia cognitiva e/ou comportamental mostrou-se eficaz na redução da ansiedade social em pacientes com transtorno de ansiedade social. No entanto, a heterogeneidade dos estudos avaliados, seja no que diz respeito a aspectos envolvendo o tratamento (duração do tratamento e protocolos utilizados) ou os pacientes avaliados (gravidade e forma de recrutamento), dificultaram a comparação dos resultados. Novos estudos de tratamento cognitivo e/ou comportamental e novas revisões envolvendo essas abordagens são necessários para melhor avaliação de nossos achados.

Descritores: Fobia social, terapia cognitiva, terapia comportamental, transtorno de ansiedade social, revisão.

\begin{abstract}
Objective: To ascertain whether cognitive and/or behavioral treatment of patients with social anxiety disorder is superior in terms of efficacy than other types of therapeutic interventions, including pharmacotherapy.

Method: Systematic analysis of all randomized clinical trials on cognitive and/or behavioral therapy vs. other forms of treatment, published until March 2009, indexed in the following databases: MEDLINE, PsycINFO, Cochrane Controlled Trials Register, LILACS and ISI Web of Science.

Results: The reported results do not suggest significant differences in the efficacy of cognitive and/or behavioral therapy and pharmacotherapy. Such treatments combined did not show higher efficacy than interventions alone. Compared with the control group, cognitive and/or behavioral therapy was superior in reducing social anxiety disorder, either in the classic model or in the newest propositions. The only exception was the social skills training, which was not effective for patients with social anxiety disorder. Cognitive and/or behavioral therapy was also superior to other psychotherapeutic interventions.

Conclusion: Cognitive and/or behavioral therapy is an effective treatment for patients with social anxiety disorder. However, the heterogeneity of the clinical trials evaluated, with reference to treatment features (time of treatment and different protocols) or characteristics of the patients (severity of their clinical picture and how they were recruited) made comparison of results between different treatments difficult. More research on cognitive and/or behavioral treatment and new reviews of these interventions are necessary to better assess our findings.
\end{abstract}

Keywords: Social phobia, cognitive therapy, behavioral therapy, social anxiety disorder, review.

Correspondência:

Sara Costa Cabral Mululo, Rua Voluntários da Pátria, 283/703, CEP 22270-000, Bairro Botafogo, Rio de Janeiro, RJ. Fax: (21) 2239.4919.

E-mail: saramululo@yahoo.com.br

Não foram declarados conflitos de interesse associados à publicação deste artigo.

Copyright (C) Revista de Psiquiatria do Rio Grande do Sul - APRS

Recebido em 31/03/2009. Aceito em 04/08/2009. 


\section{Introdução}

O transtorno de ansiedade social (TAS) é caracterizado por ansiedade excessiva frente a uma variedade de situações sociais. Os indivíduos receiam comportar-se de modo inadequado (pelo desempenho deficiente ou pela demonstração da ansiedade) e sofrer reprovação e crítica por parte de outras pessoas.

As situações sociais são evitadas ou suportadas com grande ansiedade e sofrimento por esses pacientes, embora exista o desejo de realizar o contato social temido. O comportamento fóbico-evitativo determina grave prejuízo no funcionamento do indivíduo, seja no trabalho, na escola ou nas relações sociais habituais.

A prevalência do TAS durante a vida é de $12,1 \%^{1}$ e, em amostras clínicas, os pacientes com TAS representam de 10 a $20 \%$ dos indivíduos com transtornos de ansiedade, sendo o transtorno de ansiedade mais prevalente ${ }^{2}$.

Tradicionalmente o TAS vinha recebendo pouca atenção e, até a metade da década de 1980, era considerado um transtorno negligenciado ${ }^{3}$. Foi reconhecido oficialmente como um transtorno psiquiátrico após a publicação do DSM-III em 1980. A partir daí, um número crescente de publicações tem surgido, revelando um maior interesse sobre o tema.

Apesar do volume crescente de estudos sobre TAS, ainda há questões a serem respondidas no que diz respeito a tratamentos específicos para esse transtorno, como, por exemplo, o manejo dos pacientes que não apresentam resposta aos tratamentos considerados de primeira linha e que representam cerca de 30 a $40 \%$ dos pacientes com TAS $^{4}$.

O objetivo principal deste artigo é revisar de forma sistemática os estudos controlados que avaliaram a eficácia da terapia cognitivo-comportamental (TCC) em pacientes adultos com TAS. No presente estudo, avaliamos a eficácia da TCC no TAS a partir da comparação entre TCC [técnicas comportamentais (TComp) ou terapia cognitiva (TCog)] e outros tipos de intervenção: tratamento farmacológico, outros tipos de psicoterapia e lista de espera (LE)/grupo controle.

\section{Método}

Os ensaios clínicos de tratamento cognitivo e/ou comportamental para pacientes adultos com TAS foram identificados através da busca nas seguintes bases de dados: Base ISI/Web of Science, MEDLINE, PsycINFO, Registro Cochrane de Ensaios Controlados, LILACS e em artigos relacionados. Utilizou-se a mescla das seguintes expressões nas buscas digitais: (CBT OR cognitive-behavioral therapy OR behavioral therapy OR cognitive therapy) AND (social anxiety disorder OR social phobia OR social anxiety). Ensaios clínicos controlados e randomizados publicados até março de 2009 foram incluídos sem restrição de idiomas.

Os critérios de inclusão foram: 1) estudos com participantes com diagnóstico primário de TAS segundo o DSMIII, DSM-III-R, DSM-IV ou CID-10; 2) estudos envolvendo TCog ou TComp, individual ou em grupo, comparado com tratamento farmacológico, outro tipo de psicoterapia ou LE/ grupo controle.

Na investigação realizada na MEDLINE foram localizados 377 artigos, dos quais 29 foram incluídos. No PsycINFO, de 79 estudos identificados, 14 foram separados: 10 deles em comum com MEDLINE e quatro novos. No registro Cochrane de Ensaios Controlados, foram localizados 597 estudos; 27 preenchiam os critérios de inclusão, mas todos já haviam sido encontrados nas buscas anteriores. No LILACS, dos 215 trabalhos buscados, dois preenchiam os critérios para inclusão. A base ISI/Web of Science apresentou 874 artigos, 36 já selecionados nas buscas anteriores e três inéditos. Por fim, através dos artigos relacionados, mais quatro ensaios foram incluídos. Um total de 42 artigos foi revisado.

Para fins de categorização dos resultados, considerou-se TCC quando TCog e TComp foram associadas entre si, por exemplo, terapia racional emotiva comportamental (TREC) mais exposição (EXP), reestruturação cognitiva (RC) mais EXP, TCog mais treino em tarefa de concentração (TTC) ou TCog mais treino em habilidades sociais (THS) (Figura 1).

\begin{tabular}{|ll|}
\hline Terapia cognitiva & Característica \\
\hline $\begin{array}{l}\text { Reestruturação } \\
\text { cognitiva }\end{array}$ & $\begin{array}{l}\text { Visa ajudar o paciente a identificar os pensamentos automáticos distorcidos, questionar as bases desses } \\
\text { pensamentos à luz das evidências reais (via questionamento socrático ou experimentos comportamentais) e construir } \\
\text { alternativas menos tendenciosas e padronizadas. }\end{array}$ \\
\hline $\begin{array}{l}\text { Técnicas } \\
\text { comportamentais }\end{array}$ & Características \\
\hline Exposição & $\begin{array}{l}\text { Visa expor o paciente a situações ansiogênicas gradativamente através de uma hierarquia, criada junto com o } \\
\text { paciente, até que a ansiedade naturalmente comece a diminuir (habituação). Pode ser feita através da imaginação } \\
\text { ou ao vivo. }\end{array}$ \\
\hline $\begin{array}{l}\text { Relaxamento } \\
\text { aplicado }\end{array}$ & $\begin{array}{l}\text { Visa ajudar o paciente a controlar os sintomas fisiológicos da ansiedade durante os eventos sociais. Ele aprende } \\
\text { a detectar os primeiros sinais de tensão muscular para logo assim descontrair, produzindo uma resposta contrária } \\
\text { à de ansiedade. }\end{array}$ \\
\hline $\begin{array}{l}\text { Treino em } \\
\text { habilidades sociais }\end{array}$ & $\begin{array}{l}\text { Visa ajudar o paciente a adquirir as habilidades sociais necessárias para um bom relacionamento interpessoal } \\
\text { habilidades podem ser treinadas durante a exposição. }\end{array}$ \\
\hline $\begin{array}{l}\text { Treino em tarefa } \\
\text { de concentração }\end{array}$ & $\begin{array}{l}\text { Visa ajudar o paciente a direcionar sua atenção para a tarefa em execução, e não para si mesmo, começando com } \\
\text { situações menos ansiogênicas. Com isso, ele diminui sua percepção das alterações que a ansiedade provoca em } \\
\text { seu corpo, quebrando o ciclo vicioso de aumento de ansiedade que o autofoco provoca. }\end{array}$ \\
\hline
\end{tabular}

Figura 1 - Características da terapia cognitiva e das técnicas comportamentais 


\section{Resultados}

TCC comparada ao tratamento farmacológico

TCC versus antidepressivos, benzodiazepínicos e betabloqueadores
Em 11 estudos, comparou-se a eficácia da psicoterapia (seja ela TCC, TCog, TComp isoladamente) e psicofármacos (antidepressivos, benzodiazepínicos ou betabloqueadores) no tratamento do TAS. Seis ensaios clínicos ressaltaram a superioridade da psicoterapia, três revelaram a superioridade do medicamento e dois não encontraram diferença entre as intervenções (Tabela 1).

Tabela 1 - Terapia cognitivo-comportamental comparada ao tratamento farmacológico

\begin{tabular}{|c|c|c|c|}
\hline Tratamento por estudo & $\mathbf{n}$ & $\begin{array}{c}\text { Duração } \\
\text { do tratamento } \\
\text { (semanas) }\end{array}$ & $\begin{array}{c}\text { Intervenção mais eficaz } \\
\text { (pós-teste) }\end{array}$ \\
\hline Clark $^{5}$ & & 16 & $\mathrm{TCog}$ \\
\hline TCog & 20 & & \\
\hline FLU $(20-60 \mathrm{mg} / \mathrm{d})+$ auto EXP & 20 & & \\
\hline PLO $(20-60 \mathrm{mg} / \mathrm{d})+$ auto EXP & 20 & & \\
\hline Prasko $^{6}$ & & 24 & $\mathrm{TCC}+\mathrm{PLO}$ e \\
\hline MCLO $(600 \mathrm{mg} / \mathrm{d})+$ terapia de suporte & 20 & & \\
\hline TCC + PLO & 24 & & \\
\hline MCLO + TCC & 22 & & \\
\hline Oosterbaan ${ }^{7}$ & & 15 & TCog e MCLO \\
\hline TCog & NI & & \\
\hline PLO ou MCLO (300-600 mg/d) & NI & & \\
\hline Clark $^{8}$ & & & $\mathrm{TCC}$ \\
\hline TCC + PLO & 9 & 5 & \\
\hline TCC+ BUSP & 8 & 5 & \\
\hline $\operatorname{BUSP}(15-60 \mathrm{mg} / \mathrm{d})$ & 9 & 6 & \\
\hline PLO (15-60 mg/d) & 8 & 6 & \\
\hline Turner ${ }^{9}$ & & 12 & TComp \\
\hline TComp & 21 & & \\
\hline $\operatorname{ATE}(25-100 \mathrm{mg} / \mathrm{d})$ & 21 & & \\
\hline PLO $(25-100 \mathrm{mg} / \mathrm{d})$ & 20 & & \\
\hline Mörtberg ${ }^{10}$ & & & TCog individual e em grupo \\
\hline TCog grupo intensiva & 35 & 3 & \\
\hline TCog individual & 32 & 16 & \\
\hline $\begin{array}{l}\text { ISRS (FLU 35mg/d, ou PARO } 25 \mathrm{mg} / \mathrm{d} \text {, ou MCLO 600mg/d, } \\
\text { ou CITA } 50 \mathrm{mg} / \mathrm{d} \text { ) ou BENZO (OXA } 15 \mathrm{mg} / \mathrm{d} \text { ) }\end{array}$ & 33 & 48 & \\
\hline Heimberg ${ }^{11}$ & & 12 & $\mathrm{FE}$ \\
\hline TCC grupo & 36 & & \\
\hline $\mathrm{FE}(15-75 \mathrm{mg} / \mathrm{d})$ & 33 & & \\
\hline TSE & 33 & & \\
\hline PLO(15-75 mg/d) & 31 & & \\
\hline Otto $^{13}$ & & 12 & Não houve diferença significativa entre os grupos \\
\hline CLO (4 mg/d) & 25 & & \\
\hline TCC grupo & 20 & & \\
\hline Blomhoff ${ }^{14}$ & & 24 & SERT \\
\hline SERT $(50-150 \mathrm{mg} / \mathrm{d})$ & 96 & & \\
\hline $\mathrm{SERT}+\mathrm{EXP}$ & 98 & & \\
\hline $\mathrm{EXP}+\mathrm{PLO}$ & 98 & & \\
\hline PLO (50-150 mg/d) & 95 & & \\
\hline Gelernter ${ }^{15}$ & & 12 & TCC; FE; e ALP \\
\hline TCC & 20 & & \\
\hline FE $(10-90 \mathrm{mg} / \mathrm{d})+$ INS Auto EXP & 15 & & \\
\hline $\operatorname{ALP}(0,7-6,3 \mathrm{mg} / \mathrm{d})+\mathrm{INS}$ Auto EXP & 15 & & \\
\hline PLO (de FE ou ALP) + INS Auto EXP & 15 & & \\
\hline Davidson $^{16}$ & & 14 & FLU e TCC \\
\hline FLU $(10-60 \mathrm{mg} / \mathrm{d})$ & 57 & & \\
\hline TCC grupo & 60 & & \\
\hline PLO $(10-60 \mathrm{mg} / \mathrm{d})$ & 60 & & \\
\hline FLU + TCC & 59 & & \\
\hline $\mathrm{PLO}+\mathrm{TCC}$ & 59 & & \\
\hline
\end{tabular}


Dos estudos com resultado positivo, o primeiro ${ }^{5}$ comparou 60 pacientes com TAS generalizado alocados nos grupos: TCog, fluoxetina mais EXP ou placebo mais EXP. Os resultados indicaram que o grupo de TCog foi superior aos dois tratamentos combinados no pós-teste e os ganhos foram mantidos após 12 meses.

Dois estudos avaliando a moclobemida demonstraram a eficácia tanto da TCC no pós-teste e follow-up $(1,2,15$ e 24 meses) quanto da combinação da TCC mais moclobemida. No primeiro, Prasko et al. ${ }^{6}$ avaliaram 66 pacientes com TAS generalizado utilizando três tipos de intervenções: moclobemida mais guia de suporte, moclobemida mais TCC (tratamento combinado) ou TCC mais placebo, no qual ambos grupos que continham TCC foram igualmente mais eficazes. No estudo de Oosterbaan et al. ${ }^{7}, 82$ pacientes com TAS foram alocados aleatoriamente para $\mathrm{TCog}$ ou tratamento farmacológico duplo-cego com moclobemida ou placebo, no qual o tratamento da TCog foi superior à medicação.

No estudo de Clark et al. ${ }^{8}, 34$ músicos com ansiedade de performance foram divididos em quatro braços de intervenção: buspirona, TCC em grupo mais buspirona, TCC em grupo mais placebo ou placebo. A buspirona não demonstrou ser um medicamento eficaz para o TAS quando comparada à TCC. No grupo submetido a TCC, houve redução significativa da ansiedade no pós-teste e no follow-up de 1 mês.

Os resultados da EXP por inundação foram superiores ao atenolol e placebo em uma pesquisa ${ }^{9}$ com 62 pacientes com TAS. No follow-up de 6 meses, todos os pacientes que haviam apresentado melhora, tanto os submetidos a inundação quanto os que fizeram uso do atenolol, mantiveram os benefícios.

Mortberg et al. ${ }^{10}$ avaliaram 100 pacientes randomizados para TCog intensiva de grupo, TCog individual ou tratamento com antidepressivo inibidor seletivo de recaptação de serotonina ou moclobemida ou benzodiazepínico. Após o tratamento e no follow-up de 1 ano, os resultados indicaram a TCog individual como sendo mais eficaz do que a medicação e o tratamento em grupo.

Três estudos demonstraram a eficácia superior do medicamento em relação à TCC. No primeiro ${ }^{11}$, a fenelzina demonstrou melhores resultados do que a TCC no pós-tes- te e no follow-up de 6 meses $^{12}$. Os 133 pacientes com TAS avaliados receberam TCC em grupo, fenelzina, placebo ou terapia de suporte educacional em grupo.

Em outra pesquisa, Otto et al. ${ }^{13}$ alocaram 45 indivíduos com TAS aleatoriamente para TCC em grupo ou clonazepam. Após a intervenção, todos os pacientes inicialmente randomizados tiveram, em média, igual redução da ansiedade social, embora aqueles que completaram todo o tratamento tenham experimentado maior vantagem com o clonazepam.

A sertralina demonstrou resultados contraditórios na pesquisa de Blomhoff et al. ${ }^{14}$ com 387 pacientes com TAS generalizado divididos em grupos de sertralina, sertralina mais EXP e EXP mais placebo. No pós-teste, a sertralina foi mais eficaz; porém, no follow-up de 1 ano, o grupo de EXP foi o único que demonstrou ter sustentado os ganhos terapêuticos observados durante o tratamento.

Não foi encontrada diferença entre a psicoterapia e o tratamento farmacológico em dois outros trabalhos comparando TCC com fluoxetina e com fenelzina e alprazolam. Neste último ${ }^{15}, 65$ pacientes foram divididos entre TCC em grupo, fenelzina mais instrução para EXP (ou seja, incentivo de EXP sem intervenção terapêutica), alprazolam mais instrução para EXP ou placebo mais instrução para EXP. Tanto no pós-teste quanto no follow-up de 2 meses, a TCC e os tratamentos farmacológicos foram igualmente mais eficazes do que o placebo na redução dos sintomas fóbico sociais.

Em outro ensaio clínico ${ }^{16}, 295$ pacientes com TAS generalizado foram alocados aleatoriamente em cinco grupos: fluoxetina, TCC em grupo, placebo, fluoxetina mais TCC em grupo e TCC em grupo mais placebo. Os grupos de fluoxetina e TCC sozinhos obtiveram melhoras equivalentes na redução do TAS após o tratamento, enquanto as intervenções combinadas não demonstraram vantagem adicional.

\section{TCC comparada a outros tipos de psicoterapias}

\section{TCog com THS versus terapia de suporte}

No ensaio clínico de Cottraux et al. ${ }^{17}$, o grupo de TCog mais THS foi mais eficaz do que a terapia de suporte (ou seja, escuta empática e considerações positivas sobre o discurso dos pacientes) para os 67 pacientes com TAS avaliados (Tabela 2).

Tabela 2 - Terapia cognitivo-comportamental comparada a outros tipos de psicoterapias

\begin{tabular}{|c|c|c|c|}
\hline Tratamento por estudo & $\mathbf{n}$ & $\begin{array}{c}\text { Duração } \\
\text { do tratamento } \\
\text { (semanas) }\end{array}$ & $\begin{array}{l}\text { Intervenção mais eficaz } \\
\text { (pós-teste) }\end{array}$ \\
\hline Cottraux $^{17}$ & & 12 & TCog seguida THS \\
\hline TCog seguida THS & 32 & & \\
\hline TS & 35 & & \\
\hline Taylor $^{18}$ & & 8 & RC seguida EXP \\
\hline RC individual seguida EXP em grupo & 32 & & \\
\hline TA individual seguida EXP em grupo & 28 & & \\
\hline Heimberg ${ }^{19}$ & & 12 & TCC \\
\hline TCC em grupo & 25 & & \\
\hline TSE & 24 & & \\
\hline
\end{tabular}




\section{TCog versus terapia associativa}

A eficácia da TCog foi avaliada no primeiro bloco de tratamento do estudo de Taylor et al. ${ }^{18}$, que comparou 60 pacientes com TAS divididos em dois grupos: RC individual seguida de EXP em grupo e terapia associativa individual seguida de EXP em grupo. Analisando os resultados do primeiro pós-teste (e também no follow-up de 3 meses) entre RC e terapia associativa, viu-se que o primeiro grupo demonstrou melhora significativa $\mathrm{e}$ bem superior à terapia associativa na redução dos sintomas.

\section{TCC versus terapia de suporte educacional}

A TCC foi superior à terapia de suporte educacional ${ }^{19}$ (envolvendo psicoeducação a respeito do TAS e das crenças disfuncionais e treino em assertividade sem EXP), tanto após o tratamento quanto no follow-up de 3 meses, 6 meses e 5 $\operatorname{anos}^{20}$, em estudo envolvendo 49 pacientes com TAS divididos entre TCC em grupo e terapia de suporte educacional.

\section{TCC e associações comparadas ao controle}

\section{TCC versus $\mathrm{LE}$}

Três estudos demonstraram que a TCC em grupo foi mais eficaz do que a LE na redução dos sintomas fóbicosociais. No primeiro ${ }^{21}, 31$ pacientes com TAS generalizado foram randomizados para TCC em grupo ou para a LE por 12 semanas e avaliados no pós-teste. Já o segundo ${ }^{22}$ envolveu 26 pacientes divididos em TCC de grupo intensiva (3 semanas de sessões diárias) ou LE, sendo medidos no pós-teste e no follow-up de 6 meses e de 1 ano. Por fim, no terceiro estudo $^{23}, 32$ pacientes com TAS foram divididos entre TCC em grupo ou LE, no qual o grupo de tratamento reduziu o foco da atenção em palavras e faces ameaçadoras socialmente (Tabela 3).

Tabela 3 - Terapia cognitivo-comportamental e associações comparadas a grupo controle

\begin{tabular}{|c|c|c|c|}
\hline Tratamento por estudo & $\mathbf{n}$ & $\begin{array}{c}\text { Duração } \\
\text { do tratamento } \\
\text { (semanas) }\end{array}$ & $\begin{array}{l}\text { Resultado positivo } \\
\text { no pós-teste }\end{array}$ \\
\hline Mörtberg ${ }^{22}$ & & 3 & TCC \\
\hline TCC intensiva de grupo & 13 & & \\
\hline LE & 13 & & \\
\hline D’El Rey ${ }^{21}$ & & 12 & TCC \\
\hline TCC grupo & 15 & & \\
\hline LE & 16 & & \\
\hline Pishyar $^{23}$ & & 8 & TCC \\
\hline TCC grupo & 16 & & \\
\hline LE & 16 & & \\
\hline Andersson $^{24}$ & & 9 & TCC \\
\hline TCC via internet & 32 & & \\
\hline LE & 32 & & \\
\hline Carlbring $^{25}$ & & 9 & TCC \\
\hline TCC via internet & 29 & & \\
\hline LE & 28 & & \\
\hline Titov $^{26}$ & & 10 & CACCBT \\
\hline CACCBT & 50 & & \\
\hline LE & 55 & & \\
\hline Titovev $^{28}$ & & 10 & CACCBT \\
\hline CACCBT & 43 & & \\
\hline LE & 45 & & \\
\hline $\operatorname{Titov}^{27}$ & & 10 & CACCBT \\
\hline CACCBT & 32 & & \\
\hline CCBT & 31 & & \\
\hline Controle & 35 & & \\
\hline Abramowitz $^{29}$ & & 8 & TCC via internet \\
\hline TCC via internet & 11 & & \\
\hline LE & 10 & & \\
\hline Gruber $^{32}$ & & & TCC e TCC via computador \\
\hline TCC & 18 & 12 & \\
\hline TCC assistida pelo computador & 18 & 12 & \\
\hline Controle & 18 & NI & \\
\hline Rapee $^{31}$ & & 12 & TCC; autoterapia; e autoterapia + terapeuta \\
\hline TCC grupo & 59 & & \\
\hline Autoterapia & 56 & & \\
\hline Autoterapia + terapeuta & 57 & & \\
\hline LE & 52 & & \\
\hline
\end{tabular}




\begin{tabular}{|c|c|c|c|}
\hline Hofmann ${ }^{33}$ & & 12 & TCC e EXP \\
\hline TCC & 30 & & \\
\hline EXP & 30 & & \\
\hline LE & 30 & & \\
\hline Hope $^{34}$ & & & TCC e EXP \\
\hline TCC & 28 & 12 & \\
\hline EXP & 6 & 12 & \\
\hline LE & 6 & NI & \\
\hline Stangier ${ }^{35}$ & & 15 & TCog individual e TCog em grupo \\
\hline TCog individual & 24 & & \\
\hline TCog em grupo & 26 & & \\
\hline LE & 21 & & \\
\hline Mattick $^{36}$ & & 6 & $\mathrm{EXP} ; \mathrm{RC}$ e EXP + RC \\
\hline EXP & 11 & & \\
\hline $\mathrm{RC}$ & 11 & & \\
\hline $\mathrm{EXP}+\mathrm{RC}$ & 11 & & \\
\hline LE & 10 & & \\
\hline Salaberria $^{37}$ & & & $\mathrm{EXP}$ e EXP + TCog \\
\hline EXP & 24 & 8 & \\
\hline $\mathrm{EXP}+\mathrm{TCog}$ & 24 & 24 & \\
\hline Controle & 23 & 24 & \\
\hline Clark $^{38}$ & & 14 & TCog e EXP + RA \\
\hline $\mathrm{TCog}$ & 21 & & \\
\hline $\mathrm{EXP}+\mathrm{RA}$ & 21 & & \\
\hline LE & 20 & & \\
\hline Scholing ${ }^{39}$ & & 8 & $\begin{array}{c}\text { EXP individual; TCog seguida EXP individual/ } \\
\text { grupo; e TCC individual/grupo }\end{array}$ \\
\hline EXP individual & 22 & & \\
\hline TCog seguida EXP individual/grupo & 24 & & \\
\hline TCC individual/grupo & 24 & & \\
\hline Scholing ${ }^{41}$ & & 8 & $\begin{array}{l}\text { EXP seguida TCog individual; TCog seguida } \\
\text { de EXP individual; e TCC individual }\end{array}$ \\
\hline EXP seguida TCog individual & 10 & & \\
\hline TCog seguida EXP individual & 10 & & \\
\hline TCC individual & 10 & & \\
\hline Antona $^{43}$ & & 10 & $\mathrm{EXP}+\mathrm{RC}$; EXP seguida de RC; e RC + EXP \\
\hline $\mathrm{EXP}+\mathrm{RC}$ & 31 & & \\
\hline EXP seguido RC & 23 & & \\
\hline $\mathrm{RC}+\mathrm{EXP}(\mathrm{LE})$ & 31 & & \\
\hline Bögels ${ }^{44}$ & & 8 & $\mathrm{TTC}+\mathrm{TCog}$ e RA $+\mathrm{TCog}$ \\
\hline TTC + TCog & 33 & & \\
\hline $\mathrm{RA}+\mathrm{TCog}$ & 32 & & \\
\hline LE & 65 & & \\
\hline
\end{tabular}

$\overline{\mathrm{CACCBT}}=$ clinician-assisted computerized cognitive behavioral treatment $\mathrm{CCBT}=$ computerized cognitive behavioral treatment $; \mathrm{EXP}=$ exposição; $\mathrm{LE}=$ lista de espera; $\mathrm{NI}=$ não informado; $\mathrm{PLO}=$ placebo; $\mathrm{RA}=$ relaxamento aplicado; $\mathrm{RC}=$ reestruturação cognitiva; $\mathrm{TCC}=$ terapia cognitivo-comportamental; $\mathrm{TCog}=$ terapia cognitiva; $\mathrm{TTC}=$ treino em tarefa de concentração; $\mathrm{VF}=$ video feedback .

\section{TCC via internet versus $\mathrm{LE}$}

Surgindo como alternativa para pessoas que moram em regiões onde não há tratamento específico ou para aqueles com receio de procurar ajuda, a TCC via internet demonstrou ser eficaz na redução do nível de ansiedade social em seis ensaios controlados. No primeiro, Andersson et al..$^{24}$ dividiram 64 pacientes para que fossem submetidos a um programa de TCC via internet com manual de autoajuda ou para a LE. Em outro trabalho, Carlbring et al. ${ }^{25}$ avaliaram, no pós-teste e no follow-up de 1 ano, 29 pacientes com TAS tratados com TCC via internet suplementada por telefonemas semanais com terapeuta em comparação a 28 pacientes da LE.

Já outros 105 pacientes com TAS $^{26}$ foram randomizados para um programa de TCC via internet com contato mínimo de um terapeuta por 10 semanas via e-mail ou para a LE. Tanto no pós-teste quanto no follow-up de 6 meses $^{27}$, o grupo de tratamento foi mais eficaz na melhora do humor, da habilidade e da ansiedade social.

Esse ensaio foi replicado para mais 88 pacientes $^{28}$ no qual foi encontrado no pós-teste e na avaliação de 6 meses $^{26}$ o mesmo resultado superior do programa de TCC via internet em relação ao grupo controle.

Outro programa de TCC via internet ${ }^{29}$, com contato mínimo com terapeuta ao vivo (e não via e-mail), também foi mais eficaz do que a LE para 21 pacientes com TAS leve a moderado no pós-teste de 8 semanas e na reavaliação após 3 meses.

Titov et al..$^{30}$ compararam o tratamento de TCC via internet com contato mínimo de um terapeuta via e-mail não 
só com a LE, mas também com um programa de TCC via internet sem contato terapêutico para 98 pacientes com TAS. A intervenção mais eficaz foi o grupo de TCC via internet com contato terapêutico, e não houve diferença entre o programa de TCC via internet sem terapeuta e a LE.

TCC e autoterapia (com ou sem assistência terapêutica) versus $L E$

Em pesquisa conduzida por Rapee et al. $.^{31}, 224$ pacientes com TAS generalizado grave foram submetidos a autoterapia através de um guia, autoterapia através de um guia com assistência mínima de um terapeuta e a TCC padrão. Demonstrou-se que tanto a TCC convencional quanto o tratamento alternativo de autoterapia (tratamento não presencial) foram igualmente eficazes na redução dos sintomas de ansiedade social (Tabela 3).

\section{TCC com tarefa de casa via computador versus $L E$}

A inclusão de um programa de computador para a realização da tarefa de casa no tratamento da TCC foi avaliada como eficaz em estudo realizado por Gruber et al. ${ }^{32}$. Cinquenta e quatro pacientes com TAS foram alocados randomicamente em um grupo de TCC com tarefa de casa realizada através do computador (visando preparar cognitivamente o participante antes da EXP às situações sociais temidas), um grupo de TCC com tarefa de casa sem assistência do computador e LE.

\section{TCC, TCog e EXP versus controle}

Nove ensaios clínicos controlados comprovaram a eficácia da TCC, da TCog e da EXP (aplicadas isoladamente ou associadas entre si) na redução dos sintomas fóbico-sociais quando comparadas ao grupo controle.

Dois ensaios clínicos comparando TCC, EXP e LE, um para 90 pacientes $^{33}$ e outro para 40 pacientes com TAS $^{34}$, obtiveram resultado positivo da psicoterapia na diminuição dos sintomas iniciais de ansiedade em relação à LE.

Pacientes submetidos a TCog, tanto individual quanto em grupo, obtiveram melhora significativa do TAS no pósteste em relação aos participantes da LE em estudo de Stangier et al..$^{35}$.

Em outros dois estudos, EXP e TCog obtiveram resultado positivo quando comparados à LE. No primeiro $^{36}, 43$ pacientes com TAS foram tratados com EXP, ou RC, ou EXP mais $\mathrm{RC}$ ou LE e, no segundo ${ }^{37}$, os 71 pacientes com TAS generalizada selecionados foram randomicamente divididos entre EXP, EXP mais TCog e LE.

Um novo programa de TCog e EXP associada ao relaxamento aplicado ${ }^{38}$ foi avaliado por Clark et al. e também representou melhora significativa em relação à LE em estudo envolvendo 62 pacientes com TAS.

Em dois trabalhos de Scholing et al., foram observadas diferenças significativas, após 8 semanas de tratamento e no follow-up, entre EXP, TCog seguida de EXP, EXP seguida de TCog e TCC comparadas a 4 semanas de não tratamento, considerado como grupo controle. Foram analisados 70 pacientes com TAS generalizado ${ }^{39}$ alocados em três condições: dois blocos de EXP, um bloco de TCog seguida de um bloco de EXP ou TCC integrada desde o início no pós-teste e no follow-up de 3 meses $^{40}$. No segundo ensaio clínico, 30 pacientes $^{41}$ com TAS com queixa principal de suar, tremer e ruborizar foram divididos em TCog seguida de EXP, EXP seguida de TCog ou TCC integrada desde o início no pósteste e no follow-up de 18 meses $^{42}$.

Oitenta e cinco pacientes com TAS foram randomizados para o grupo de EXP + RC, ou para EXP seguida de RC, ou para RC seguida de $\operatorname{EXP}^{43}$ (que 3 meses antes foi grupo controle). Os resultados confirmaram que as três intervenções são igualmente eficazes na redução do TAS, isto é, tanto o tratamento cognitivo e comportamental simultâneo (EXP + $\mathrm{RC})$ quanto ambos tratamentos aplicados em separado em momentos diferentes (EXP seguido de RC e RC seguida de EXP) são superiores ao grupo controle.

\section{TTC com TCog versus LE}

Bogels et al. ${ }^{44}$ avaliaram 65 pacientes com TAS com queixa principal de suar, tremer, ruborizar e congelar em público divididos randomicamente em grupos de TTC seguido de TCog ou relaxamento seguido de TCog. A associação da TCog a outras TComp (não somente EXP) demonstrou ser eficaz na redução do TAS comparado ao período de espera.

\section{TComp comparada à $\mathbf{L E}$}

EXP e associações versus $L E$

As TComp apresentaram resultado positivo no tratamento do TAS em relação à LE em dois estudos. No primei$\mathrm{ro}^{45}$, avaliando 36 pacientes com medo de falar em público, os resultados do pós-teste indicaram a superioridade da EXP sobre a LE. No segundo ${ }^{46}, 31$ pacientes com TAS com medo de ruborizar foram divididos randomicamente em um grupo de EXP, de TTC ou LE (Tabela 4).

A EXP associada a outras técnicas demonstrou ser mais eficaz do que a LE no tratamento dos sintomas fóbico-sociais em três ensaios clínicos controlados. No primeiro, Butler et al. ${ }^{47}$ alocaram 45 pacientes em três grupos: EXP com manejo da ansiedade, EXP com intervenção não-específica ou LE. Em outro ensaio, Mersch et al. ${ }^{48}$ dividiram 34 pacientes aleatoriamente em três grupos: EXP, tratamento integrado (TREC mais THS e EXP) e LE. No último ${ }^{49}, 30$ estudantes universitárias com TAS, com queixa principal de medo de falar em público, foram designadas aleatoriamente para um grupo de EXP mais treinamento em autoinstrução e respiração ou para um grupo controle. Tanto no pós-teste quanto no follow-up de 2 anos, os participantes da EXP apresentaram maior diminuição da resposta de ansiedade do que o grupo controle.

Somente no pós-teste do trabalho de Smits et al. ${ }^{50}$ - no qual 77 pacientes com TAS foram divididos em EXP, tratamento placebo, EXP com video feedback da performance do paciente e EXP com video feedback da resposta da plateia que assistia a EXP - os três tratamentos obtiveram melhora equivalente na redução do TAS em relação ao placebo. Porém, no 
Tabela 4 - TComp comparada à lista de espera

\begin{tabular}{|c|c|c|c|}
\hline Tratamento por estudo & n & $\begin{array}{c}\text { Duração } \\
\text { do tratamento } \\
\text { (semanas) }\end{array}$ & $\begin{array}{l}\text { Resultado positivo } \\
\text { no pós-teste }\end{array}$ \\
\hline Newman ${ }^{45}$ & & 8 & EXP \\
\hline $\begin{array}{l}\text { EXP } \\
\text { LE }\end{array}$ & $\begin{array}{l}18 \\
18\end{array}$ & & \\
\hline Olivares $^{49}$ & & 6 & TComp \\
\hline TComp & 14 & & \\
\hline $\begin{array}{l}\text { Controle } \\
\text { Smits }^{50}\end{array}$ & 15 & EX & EXP; EXP + VF da performance; e EXP + VF do público \\
\hline PLO & 15 & & \\
\hline EXP & 23 & & \\
\hline $\mathrm{EXP}+\mathrm{VF}$ da performance & 19 & & \\
\hline EXP + VF do público & 20 & & \\
\hline Butler $^{47}$ & & 7 & $\mathrm{EXP}+\mathrm{MA} \mathrm{e} \mathrm{EXP} \mathrm{+} \mathrm{TNE}$ \\
\hline $\mathrm{EXP}+\mathrm{MA}$ & 15 & & \\
\hline $\mathrm{EXP}+\mathrm{TNE}$ & 15 & & \\
\hline LE & 15 & & \\
\hline $\operatorname{Mersch}^{48}$ & & 14 & EXP e EXP + TREC + THS \\
\hline EXP & NI & & \\
\hline Tratamento integrado (EXP + TREC + THS) & NI & & \\
\hline LE & NI & & \\
\hline Mulken $^{46}$ & & 6 & EXP e TTC \\
\hline EXP & 12 & & \\
\hline TTC & 14 & & \\
\hline LE & 13 & & \\
\hline Stravynski $^{51}$ & & 24 & Não houve diferença significativa entre os grupos \\
\hline THS + INTER & 32 & & \\
\hline Tratamento não específico + INTER & 36 & & \\
\hline
\end{tabular}

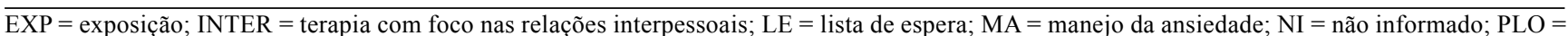
placebo; TComp = técnicas comportamentais; THS = treino em habilidades sociais; $\mathrm{TNE}=$ tratamento não específico; TREC $=$ terapia racional emotiva; $\mathrm{TTC}=$ treino em tarefa de concentração; $\mathrm{VF}=$ video feedback.

follow-up de 1 mês essa vantagem não se manteve e os grupos de intervenção com EXP obtiveram resultado negativo em relação ao grupo placebo. A duração do tratamento nesse estudo foi de 1 semana.

\section{THS versus tratamento não-específico}

O THS, avaliado na pesquisa de Stravynski et al..$^{51} \mathrm{com}$ 68 participantes com TAS, não obteve resultado diferente no pós-teste e no follow-up de 1 ano no funcionamento social dos pacientes com TAS quando comparado ao tratamento não-específico sem THS ou ao período anterior à intervenção considerado como LE.

\section{Discussão}

Os estudos controlados avaliados apontam para a eficácia das intervenções cognitivas e comportamentais (tais como EXP e TTC), sozinhas ou associadas a outras técnicas (relaxamento e treino em respiração), na redução dos sintomas do $\mathrm{TAS}^{17-19}$ quando comparadas a outros tipos de abordagens psicoterápicas ou a grupo controle 21-26,28-34,41,43. $^{2}$. Tais achados são confirmados por Acarturk et al. ${ }^{52} \mathrm{em}$ estudo meta-analítico recente que afirmou também que essa eficácia tende a se manter e, em alguns casos, até melhorar nas medidas de follow-up. Contudo, na análise entre os subgrupos que incluíam RC, EXP, THS e relaxamento aplicado, não foram encontrados altos effect sizes, pois a maioria dos ensaios avaliados misturava essas técnicas e outros poucos as examinaram isoladamente.

A EXP demonstrou eficácia significativa tanto sozinha ${ }^{45}$ quanto associada a outros recursos como manejo da ansieda$\mathrm{de}^{43,44}$, THS e TREC ${ }^{48}$. Em apenas um estudo ${ }^{50}$ houve resultado negativo, o que poderia ser explicado pela curta duração do tratamento instituído. Enquanto nesse estudo a intervenção terapêutica durou 1 semana, nos ensaios nos quais foram obtidos resultados positivos ${ }^{45-50}$ os pacientes foram submetidos a, no mínimo, 6 semanas de tratamento.

A exceção dentre as TComp estudadas foi o THS que, sozinho, não demonstrou eficácia significativa na redução dos sintomas fóbico-sociais ${ }^{51}$. Quando associado à $\mathrm{TCog}{ }^{17}$ ou à EXP ${ }^{48}$, o THS mostrou-se eficaz, apontando para a necessidade de novos ensaios clínicos que permitam melhor avaliação da eficácia dessa intervenção.

As novas formas de apresentação da TCC, como a autoterapia através de um livro-guia ${ }^{31}$, a terapia via internet ${ }^{24-26,28-30} \mathrm{e}$ 
a realização da tarefa de casa auxiliada por programa de computador $^{32}$, surgem como alternativas promissoras no tratamento do TAS. A prática da TCC envolve terapeutas habilidosos e treinados, nem sempre disponíveis, além de custos de transporte e honorários. Nesse sentido, essas intervenções representariam importantes alternativas aumentando a acessibilidade e a disponibilidade do tratamento.

A comparação entre o tratamento farmacológico e a TCC realizada em alguns ensaios clínicos não conduz a resultado conclusivo. Ambos foram eficazes na maioria dos estudos avaliados e, apesar de alguns resultados apontarem a superioridade da TCC frente aos fármacos, os dados avaliados não permitem a generalização desses achados.

Os resultados foram inconsistentes em alguns estudos. Dois estudos envolvendo a fenelzina, por exemplo, apresentaram resultados opostos, havendo superioridade em relação à TCC em um estudo ${ }^{11}$ e não havendo diferença significativa entre os dois em outro estudo ${ }^{15}$. Situação semelhante foi observada com a fluoxetina, que apresentou eficácia inferior à TCog e EXP em um ensaio clínico ${ }^{5}$ e foi tão eficaz quanto à TCC em outro ensaio ${ }^{15}$. Houve, no caso das duas substâncias, diferença entre as doses de medicação empregadas que, associada à heterogeneidade das intervenções cognitivas e comportamentais utilizadas, dificultaram a avaliação dos resultados. Novos ensaios clínicos controlados são necessários para melhor avaliação dessas intervenções.

Ao contrário do que se preconiza na prática clínica, não há evidência clara de maior eficácia do tratamento combinado, TCC mais medicamento ou TCog mais TComp, quando comparado a essas intervenções isoladas. A associação mostrou-se menos eficaz do que a $\mathrm{TCog}^{5}$, a $\mathrm{EXP}^{14}$, e a TCC e fluoxetina ${ }^{16}$ empregadas separadamente. Em outro ensaio clínico, a combinação de terapia e medicação foi tão eficaz quanto a $\mathrm{TCC}^{6}$. Importante questionar porque esses resultados científicos se mostram tão distantes, por ora opostos, ao que se observa na prática clínica do tratamento desse transtorno, uma vez que é cada vez mais recomendado aos clínicos que busquem subsídios e atualização da sua prática terapêutica nos resultados dos ensaios clínicos controlados de qualidade publicados em sua área de atuação.

Nos estudos avaliando o uso de medições no follow-up, foram adotados intervalos diferentes de seguimento, variando de 1 mês $^{8}$ a 5 anos $^{20}$, o que torna difícil a análise comparativa dos resultados. Não há consenso sobre o tempo ideal de tratamento no TAS, o que dificulta a avaliação adequada a respeito da manutenção dos benefícios adquiridos.

O número e a duração das sessões variava nos diferentes protocolos de tratamento. A gravidade do TAS também diferia entre os estudos: enquanto em alguns ensaios os pacientes selecionados apresentavam gravidade leve a moderada ${ }^{29}$, em outros $^{6}$ o subtipo generalizado grave do TAS era o critério de inclusão. A forma de recrutamento dos pacientes também diferia entre os estudos, excluindo muitas vezes pacientes com TAS que não buscaram ajuda, potencialmente mais graves e com maiores limitações. Tamanha heterogeneidade dos estudos selecionados dificulta a comparação dos resultados e não permite conclusões consistentes a respeito da eficácia das intervenções terapêuticas avaliadas.

Nossa revisão sistemática, por ser descritiva, possui algumas limitações metodológicas que não permitem a generalização dos resultados e apontam para a necessidade de novas investigações para uma avaliação mais precisa da eficácia das diferentes intervenções descritas.

\section{Conclusão}

Os dados avaliados apontam para a eficácia das diferentes técnicas de TCC no TAS; no entanto, algumas questões permanecem sem resposta. Um percentual significativo de pacientes não responde às intervenções propostas, ou apresentam sintomas residuais e há carência de estudos investigando essa população. A investigação cuidadosa das características dos pacientes resistentes e os possíveis preditores de resposta ao tratamento precisam ser mais bem elucidados.

Apesar do número crescente de estudos investigando as TCC no tratamento do TAS, há hiatos a serem discutidos no tratamento desses pacientes. Novos ensaios clínicos com protocolos de intervenção menos heterogêneos, medidas de avaliação padronizadas e revisões comparando técnicas específicas de TCC são extremamente relevantes para a melhor compreensão desse cenário. Além disso, novas revisões sistemáticas utilizando técnicas de avaliação meta-analíticas permitiriam comparação mais precisa das intervenções avaliadas e maior esclarecimento dos resultados.

Considerando que a TCC não se reduz às suas técnicas, elas são o início de uma investigação que nos permite examinar cuidadosamente como o transtorno afeta diferentemente cada sujeito, a fim de expandir nossa conceituação acerca do seu modo de pensar, sentir e agir.

\section{Referências}

1. Kessler RC, Chiu WT, Demler O, Merikangas KR, Walters EE. Prevalence, severity, and comorbidity of 12-month DSM-IV disorders in the national Comorbidity Survey replication. Arch Gen Psychiatry. 2005;62(6):617-27.

2. Kessler RC, McGonagle KA, Zhao S, Nelson CB, Hughes M, Eshleman S, et al. Lifetime and 12-month prevalence of DSM-III-R psychiatric disorders in the United States: results from the National Comorbidity Survey. Arch Gen Psychiatry. 1994;51(1):8-19.

3. Liebowitz MR, Gorman JM, Fyer AJ, Klein DF. Social phobia. Review of a neglected anxiety disorder. Arch Gen Psychiatry. 1985;42(7):729-36.

4. de Menezes GB, Fontenelle LF, Mululo S, Versiani M. Resistência ao tratamento nos transtornos de ansiedade: fobia social, transtorno de ansiedade generalizada e transtorno do pânico. Rev Bras Psiquiatr. 2007;29(Suppl II):S55-60.

5. Clark DM, Ehlers A, McManus F, Hackmann A, Fennell M, Campbell H, et al. Cognitive therapy versus fluoxetine in generalized social phobia: a randomized placebo-controlled trial. J Consult Clin Psychol. 2003;71(6):1058-67.

6. Prasko J, Dockery C, Horácek J, Houbová P, Kosová J, Klaschka J, et al. Moclobemide and cognitive behavioral therapy in the treatment of social phobia. A six-month controlled study and 24 months follow up. Neuro Endocrinol Lett. 2006;27(4):473-81.

7. Oosterbaan DD, van Balkom AJ, Spinhoven P, van Oppen P, van Dyck R. Cognitive therapy versus moclobemide in social phobia: a controlled study. Clin Psychol Psychother. 2001;8(4):263-73. 
8. Clark DB, Agras WS. The assessment and treatment of performance anxiety in musicians. Am J Psychiatry. 1991;148(5):598-605.

9. Turner SM, Beidel DC, Jacob RG. Social phobia: a comparison of behavior therapy and atenolol. J Consult Clin Psychol. 1994;62(2):350-8.

10. Mörtberg E, Clark DM, Sundin O, Aberg Wistedt A. Intensive group cognitive treatment and individual cognitive therapy vs. treatment as usual in social phobia: a randomized controlled trial. Acta Psychiatr Scand. 2007;115(2):142-54.

11. Heimberg RG, Liebowitz MR, Hope DA, Schneier FR, Holt CS, Welkowitz LA, et al. Cognitive behavioral group therapy vs. phenelzine therapy for social phobia: 12-week outcome. Arch Gen Psychiatry. 1998;55(12):1133-41.

12. Liebowitz MR, Heimberg RG, Schneier FR, Hope DA, Davies S, Holt CS, et al. Cognitive-behavioral group therapy versus phenelzine in social phobia: longterm outcome. Depress Anxiety. 1999;10(3):89-98.

13. Otto MW, Pollack MH, Gould RA, Worthington JJ 3rd, McArdle ET, Rosenbaum JF. A comparison of the efficacy of clonazepam and cognitive-behavioral group therapy for the treatment of social phobia. J Anxiety Disord. 2000;14(4):345-58.

14. Blomhoff S, Haug TT, Hellström K, Holme I, Humble M, Madsbu HP, et al. Randomised controlled general practice trial of sertraline, exposure therapy and combined treatment in generalized social phobia. Br J Psychiatry. 2001;179:23-30.

15. Gelernter CS, Uhde TW, Cimbolic P, Arnkoff DB, Vittone BJ, Tancer ME, et al. Cognitive-behavioral and pharmacological treatments of social phobia. A controlled study. Arch Gen Psychiatry. 1991;48(10):938-45.

16. Davidson JR, Foa EB, Huppert JD, Keefe FJ, Franklin ME, Compton JS, et al. Fluoxetine, comprehensive cognitive behavioral therapy, and placebo in generalized social phobia. Arch Gen Psychiatry. 2004;61(10):1005-13.

17. Cottraux J, Note I, Albuisson E, Yao SN, Note B, Mollard E, et al. Cognitive behavior therapy versus supportive therapy in social phobia: a randomized controlled trial. Psychother Psychosom. 2000;69(3):137-46.

18. Taylor S, Woody S, Koch WJ, McLean P, Paterson RJ, Anderson KW. Cognitive restructuring in the treatment of social phobia. Efficacy and mode of action. Behav Modif. 1997;21(4):487-511

19. Heimberg RG, Dodge CS, Hope DA, Kennedy CR, Zollo LJ, Becker RE. Cognitive behavioral group treatment for social phobia: Comparison with a credible placebo control. Cogn Ther Res. 1990;14(1):1-23.

20. Heimberg RG, Salzman DG, Holt CS, Blendell KA. Cognitive-behavioral group treatment for social phobia: Effectiveness at five-year follow up. Cogn Ther Res. 1993;17(4):325-39.

21. D’El Rey GJ, Lacava JP, Cejkinski A, Mello SL. Tratamento cognitivo-comportamental de grupo na fobia social: resultados de 12 semanas. Rev Psiquiatr Clin. 2008;35(2):79-83.

22. Mörtberg E, Karlsson A, Fyring C, Sundin O. Intensive cognitive-behavioral group treatment (CBGT) of social phobia: a randomized controlled study. J Anxiety Disord. 2006;20(5):646-60.

23. Pishyar R, Harris LM, Menzies RG. Responsiveness of measures of attentional bias to clinical change in social phobia. Cogn Emot. 2008;22(7):1209-27.

24. Andersson G, Carlbring P, Holmström A, Sparthan E, Furmark T, Nilsson-Ihrfelt E, et al. Internet-based self-help with therapist feedback and in vivo group exposure for social phobia: a randomized controlled trial. J Consult Clin Psychol. 2006;74(4):677-86.

25. Carlbring P, Gunnarsdóttir M, Hedensjö L, Andersson G, Ekselius L, Furmark T. Treatment of social phobia: randomised trial of internet-delivered cognitivebehavioral therapy with telephone support. Br J Psychiatry. 2007;190:123-8.

26. Titov N, Andrews G, Schwencke G, Drobny J, Einstein D. Shyness 1: distance treatment of social phobia over the Internet. Aust N Z J Psychiatry. 2008;42(7):585-94.

27. Titov N, Andrews G, Johnston L, Schwencke G, Choi I. Shyness programme: longer term benefits, cost-effectiveness, and acceptability. Aust N Z J Psychiatry. 2009;43(1):36-44

28. Titov N, Andrews G, Schwencke G. Shyness 2: treating social phobia online: replication and extension. Aust N Z J Psychiatry. 2008;42(7):595-605.
29. Abramowitz JS, Moore EL, Braddock AE, Harrington DL. Self-help cognitivebehavioral therapy with minimal therapist contact for social phobia: a controlled trial. J Behav Ther Exp Psychiatry. 2009;40 (1): 98-105.

30. Titov N, Andrews G, Choi I, Schwencke G, Mahoney A. Shyness 3: randomized controlled trial of guided versus unguided Internet-based CBT for social phobia. Aust N Z J Psychiatry. 2008;42(12):1030-40.

31. Rapee RM, Abbott MJ, Baillie AJ, Gaston JE. Treatment of social phobia through pure self help and therapist-augmented self help. Br J Psychiatry. 2007;191:246-52.

32. Gruber K, Moran PJ, Roth WT, Taylor CB. Computer-assisted cognitive behavioral group therapy for social phobia. Behav Ther. 2001;32(1):155-65.

33. Hofmann SG, Moscovitch DA, Kim HJ, Taylor AN. Changes in self-perception during treatment of social phobia. J Consult Clin Psychol. 2004;72(4):588-96.

34. Hope DA, Heimberg RG, Bruch MA. Dismantling cognitive-behavioral group therapy for social phobia. Behav Res Ther. 1995;33(6):637-50.

35. Stangier U, Heidenreich T, Peitz M, Lauterbach W, Clark DM. Cognitive therapy for social phobia: individual versus group treatment. Behav Res Ther. 2003;41(9):991-1007.

36. Mattick R, Peters L, Clarke J. Exposure and cognitive restructuring for social phobia: a controlled study. Behav Ther. 1989;20(1):3-23.

37. Salaberria K, Echeburua E. Long-term outcome of cognitive therapys contribution to self-exposure in vivo to the treatment of generalized social phobia. Behav Modif. 1998;22(3):262-84.

38. Clark DM, Ehlers A, Hackmann A, McManus F, Fennell M, Grey N, et al. Cognitive therapy versus exposure and applied relaxation in social phobia: A randomized controlled trial. J Consult Clin Psychol. 2006;74(3):568-78.

39. Scholing A, Emmelkamp PM. Exposure with and without cognitive therapy for generalized social phobia: effects of individual and group treatment. Behav Res Ther. 1993;31(7):667-81.

40. Scholing A, Emmelkamp PM. Treatment of generalized social phobia: results at long-term follow-up.Behav Res Ther. 1996;34(5-6):447-52.

41. Scholing A, Emmelkamp PM. Cognitive and behavioural treatments of fear of blushing, sweating or trembling. Behav Res Ther. 1993;31(2):155-70.

42. Scholing A, Emmelkamp PM. Treatment of fear of blushing, sweating, or trembling. Results at long-term follow-up. Behav Modif. 1996;20(3):338-56.

43. Antona CJ, García-López LJ. Repercusión de la exposición y reestructuración cognitiva sobre la fobia social. Rev Latinoam Psicol. 2008;40(2):281-92.

44. Bögels SM. Task concentration training versus applied relaxation, in combination with cognitive therapy, for social phobia patients with fear of blushing, trembling, and sweating. Behav Res Ther. 2006;44(8):1199-210.

45. Newman MG, Hofmann SG, Trabert W, Roth W, Taylor CB. Does behavioral treatment of social phobia lead to cognitive changes? Behav Ther. 1994;25(3):503-17.

46. Mulken S, Bögels SM, de Jong PJ, Louwers J. Fear of blushing: effects of task concentration training versus exposure in vivo on fear and physiology. J Anxiety Disord. 2001;15(5):413-32.

47. Butler G, Cullington A, Munby M, Amies P, Gelder M. Exposure and anxiety management in the treatment of social phobia. J Consult Clin Psychol. 1984;52(4):642-50.

48. Mersch PP. The treatment of social phobia: the differential effectiveness of exposure in vivo and an integration of exposure in vivo, rational emotive therapy and social skills training. Behav Res Ther. 1995;33(3):259-69.

49. Olivares J, Garcia Lopez LJ. Resultados a largo plazo de un tratamiento en grupo para el miedo a hablar en publico. Psicothema. 2002;14(2):405-9.

50. Smits JA, Powers MB, Buxkamper R, Telch MJ. The efficacy of videotape feedback for enhancing the effects of exposure-based treatment for social anxiety disorder: a controlled investigation. Behav Res Ther. 2006;44(12):1773-85.

51. Stravynski A, Arbel N, Bounader J, Gaudette G, Lachance L, Borgeat F, et al. Social phobia treated as a problem in social functioning: a controlled comparison of two behavioral group approaches. Acta Psychiatr Scand. 2000;102(3):188-98.

52. Acarturk C, Cuijpers P, van Straten A, de Graaf R. Psychological treatment of social anxiety disorder: a meta-analysis. Psychol Med. 2008;39(2):241-54. 